\title{
Quality Optimization of Micro-Hole In Laser Drilling
}

\author{
B. B. Satapathy ${ }^{1}$, J. Rana ${ }^{2}$, and K. P. Maity ${ }^{3}$ \\ ${ }^{1}$ Asst. Professor, Mech. Engg. Department, P.K.A.College of Engg. Bargarh-768028. \\ ${ }^{2}$ Professor, Mech. Engg. Deparment, VSSUT, Burla Sambalpur, -768018 \\ ${ }^{3}$ Professor, Mech. Engg. Department, NIT, Rourkela -769008
}

\begin{abstract}
Laser drilling is a popular non-traditional machining technique for producing large numbers of cooling holes of various sizes $(<1 \mathrm{~mm})$ and angles in modern aerospace gas turbine components such as turbine blades, nozzle guide vanes, combustion chambers and after burners. Though the rate of production of micro-hole (i.e. productivity) is very high but the quality of hole (such as straightness, circularity, HAZ) is very poor due to unique nature of the process. In the present study, SEM analysis is carried out on the micro-holes produced on a medium carbon steel specimen based on Taguchi's orthogonal array and finally recommendation is made for optimum selection of process parameter such as: pulse frequency, pulse width and assist gas flow rate for getting a good quality hole.
\end{abstract}

Key words: $\quad$ Laser drilling, hole quality, Taguchi method, optimization, SEM analysis.

\section{Introduction}

The word LASER is an acronym for "Light Amplification by Stimulated Emission of Radiations". A laser system or simply a laser is an electro-optical device used to convert electrical energy into electromagnetic energy in the form of a beam of light. Since last three decades, lasers have been used as potential tools for materials processing due to the unique properties such as: coherent, monochromatic beam of low divergence and having high energy density. Among various applications, laser drilling is a popular process for producing large numbers of cooling holes of various sizes and angles [1] in the modern aerospace gas turbine components such as turbine blades, nozzle guide vanes, and combustion chambers and after burners: Besides these applications, it has become a fast growing method in automobile, electronic and pharmaceutical Industries. Laser is an attractive tool because of the following advantages [2] over mechanical methods such as (i) ability to drill ceramics, high strength materials and composites(ii) higher accuracies and smaller dimensions (.018 -1.mm) (iii) high productivity (i.e. more than 100 holes/s) without tool changing and (iv) ability to drill inaccessible areas. Generally low to medium power pulsed Nd-YAG laser with Gaussian beam $\left(\mathrm{TEM}_{00}\right)$ mode is suitable for laser drilling. Further, the position of the work piece should be at focal plane of the lens. It facilitates least beam diameter, high energy density and the holes become straighter compared to other positions.

The mechanism of the laser drilling process is explained with the help of the Fig.1.
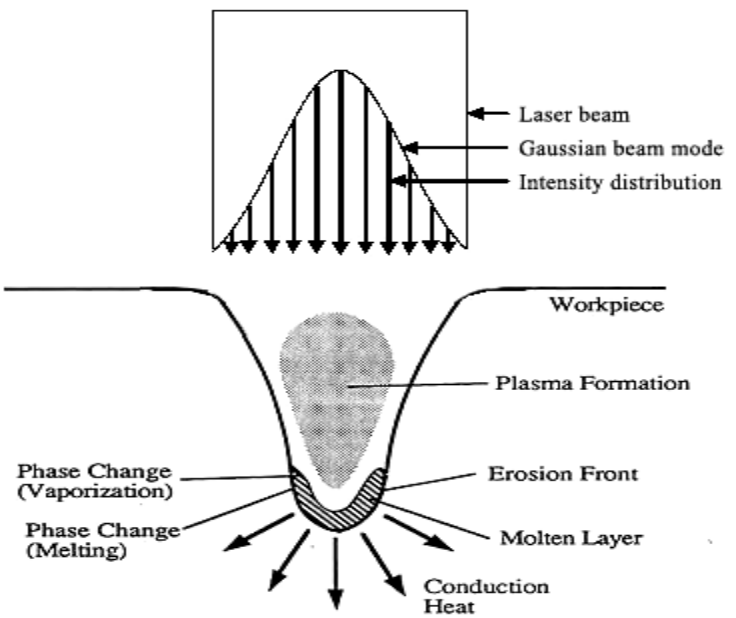

Fig. 1 : Mechanism of Laser Drilling.

When a laser beam is focused in to the workpiece surface, a portion of the beam is absorbed on the surface and some part is reflected back which depends upon the nature of surface finish and wave length of laser beam. The photons of absorbed laser beam interact with the outer most atom of the workpiece surface and heat is developed. Because of the focused and high energy density of the incident laser beam, a portion of the workpiece material gets melted and vaporized. In principle, laser drilling is governed by an energy balance between the irradiating energy from the laser beam and the 
conduction of heat into the workpiece, the energy losses to the environment and the energy required for phase change in the workpiece [3]

In laser drilling the significant mechanism of material removal is the melt ejection [4]. Vapourization within the hole creates high pressure gradient as a result of which molten material is expelled out from the hole. Because of melting and vaporization processes [5], the hole becomes tapered and bell shaped at the top. In order to protect the lens and sometimes to accelerate the drilling process, assist gases like air, oxygen, nitrogen or argon is used. Laser drilled holes are associated with the deposition of spatters because, the ejected materials resolidifies and adheres around the periphery of the holes [6].

It is obvious from the aforesaid discussion that though the process is capable of producing large numbers of fine holes on any hard and brittle material on one hand, the holes become tapered associated with oxide layers and or heat affected zone, spatters deposition on the surface, lack of circularity, presence of crack and shallow depth on the other hand. In view of the above facts, researchers are trying to minimize these defects to make this process more attracting. The main parameters which govern the quality of the hole are: pulse width, pulse frequency, power density, type and flow rate of assist gases.

Low et al [7] did parametric study for the minimization of spatter deposition. Later, Zhu et al [8] developed a new micromachining technique using a mixed mode ablation approach to minimize the common problems such as HAZ, recast and microcracks. Guo et al [9] develop a novel laser drilling method based on gel - casting technology to minimize spatter and microcracks. Wang et al [10] experimentally studied the effect of assist gases such as oxygen, argon and nitrogen on the micro structures of recast layer and reported that these are independent of the type of assist gases. Schoonderbeek et al [11] studied the influence of pulse width on the quality of hole using a excimer laser. Peter et al [12] studied the drilling rate, quality of hole and the effect related to the plasma formation on an AIN ceramics using nanosecond pulses of Nd-YAG laser. Nedialkov et al [13] experimentally investigated the influence of laser parameters and material properties on the drilling rate, quality of hole and plasma formation. Later Campbell et al [14] analysed the laser drilling parameters for the optimization of shallow hole drilling using a factorial design of experiment approach. Naeem Mohammed [15] investigated the effect of various laser and processing parameters on laser drilling rates, recast layer, taperness, oxide layer and cracking using coated and uncoated Ni- based supper alloys. Recently Rana et al [16] experimentally studied the effect of pulse frequency, pulse width and power on the aspect ratio and heat affected zone on a mild steel specimen without using optimization technique. The present paper deals with experimental investigation of laser and process parameters using SEM analysis for quality optimizations such as hole circularities, heat affected zone and aspect ratio, spatter deposition on a medium carbon steel specimen. In order to make the costly experiment economical, the experiments are planned as per Taguchi's L $\mathrm{L}_{9}$ orthogonal array.

\section{Experimentation}

\subsection{Descriptions of Experiment}

A pulsed Nd-YAG laser system was used for the experimental work with the following specifications.

(i) Rated average power $=100 \mathrm{~W}$

(ii) Rated maximum pulse energy $=2$ joules

(iii) Wave length $=1.06 \mu \mathrm{m}$

(iv) Pulse width $=300 \mu$ s to $1000 \mu \mathrm{s}$

(v) Pulse frequency $=1,2,3$ pulses $/ \mathrm{sec}$.

Arrangement was made to regulate the flow of assist gas. A specimen was prepared from medium carbon steel with dimension $100 \mathrm{~mm} \times 10 \mathrm{~mm} \times 8 \mathrm{~mm}$. During the process of drilling it was kept on a platform positioned at the focal plane of the lens. The surface of interest was ground with a surface grinding machine with surface roughness (Ra) of $2 \mu \mathrm{m}$. A set of 9 different holes were drilled on one longitudinal surface of the specimen following Taguchi's L 9 orthogonal array. Three controllable parameters such as pulse width, pulse frequency and air flow rate were varied as presented in Table 1 .

Table -1 : Controllable Parameters and their levels

\begin{tabular}{|l|l|l|l|l|l|}
\hline $\begin{array}{c}\text { Sl. } \\
\text { No. }\end{array}$ & \multicolumn{1}{|c|}{ Controllable Parameters } & Unit & $\begin{array}{c}\text { Low } \\
\text { level }\end{array}$ & $\begin{array}{c}\text { Medium } \\
\text { level }\end{array}$ & \multicolumn{1}{|c|}{$\begin{array}{c}\text { High } \\
\text { level }\end{array}$} \\
\hline 1 & Pulse width & $\mu \mathrm{s}$ & 500 & 700 & 900 \\
\hline 2. & Pulse frequency & $\mathrm{s}^{-1}$ & 1 & 2 & 3 \\
\hline 3. & Air flow rate & $\mathrm{mm}^{3} \mathrm{~s}^{-1}$ & $0.8 \times 10^{5}$ & $2.5 \times 10^{5}$ & $4.1 \times 10^{5}$ \\
\hline
\end{tabular}


IOSR Journal of Engineering

Mar. 2012, Vol. 2 (3) pp: 382-388

\subsection{Experimental Results}

After the drilling operation was over, the different parameters such as hole diameter, thickness of heat affected zone, approximate depth etc. were measured under a scanning electron microscope. The top views of few micro-holes are presented in Figures 2 to 4.

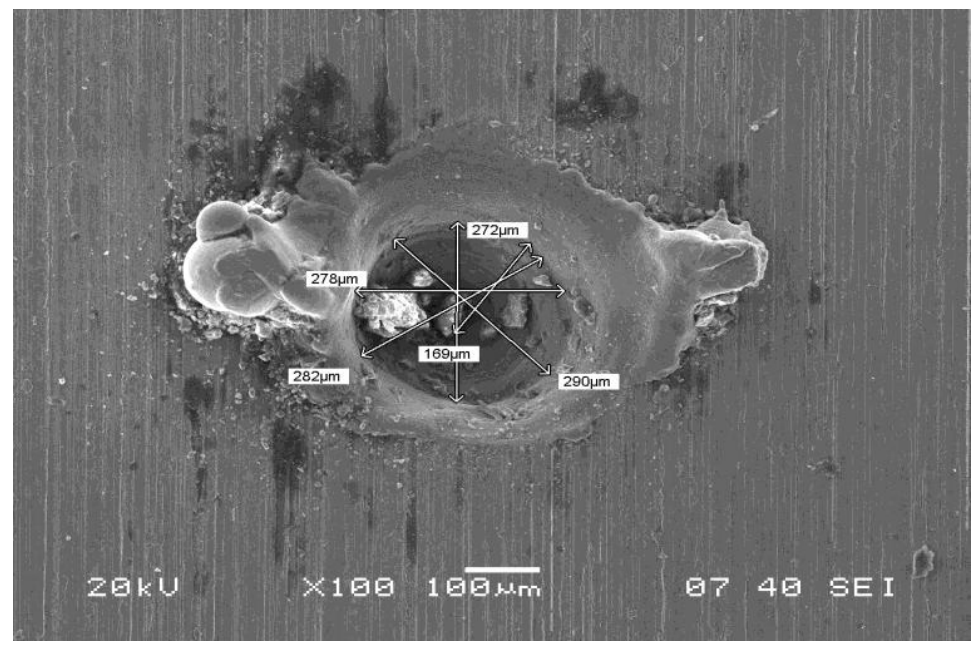

Fig. 2 : SEM photograph at process parameters : pulse width $=500 \mu \mathrm{s}$, number of pulse per second $=3$, average power $=1.7 \mathrm{~W}$, air flow rate $=4.1 \times 10^{5} \mathrm{~mm}^{3} / \mathrm{s}$, time $=20 \mathrm{sec}$.

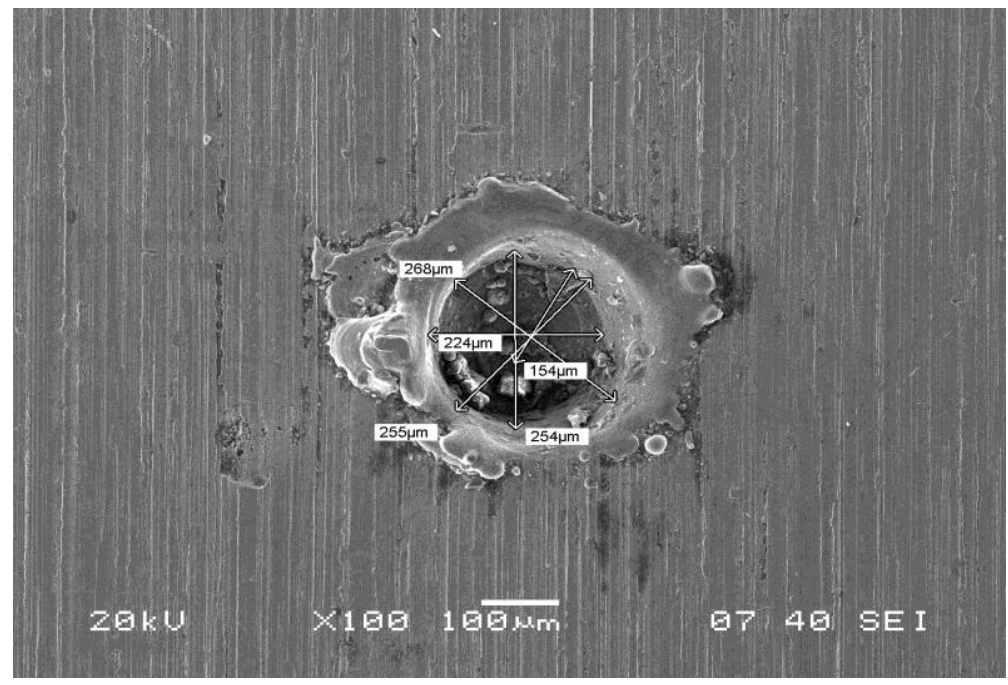

Fig. 3: SEM photograph at process parameters: pulse width $=700 \mu$ s, number of pulse per second $=3$, average power $=2 \mathrm{~W}$, air flow rate $=0.8 \times 10^{5} \mathrm{~mm}^{3} / \mathrm{s}$, time $=20 \mathrm{sec}$. 


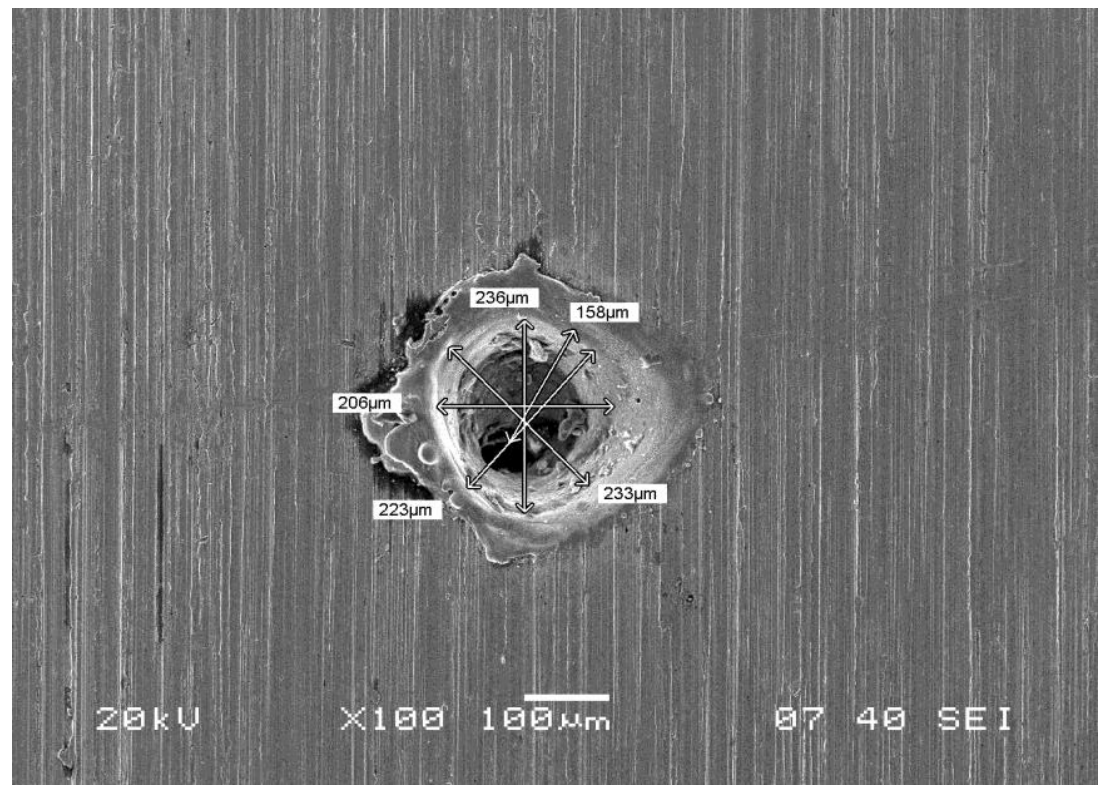

Fig. 4: SEM photograph at process parameters : pulse width $=900 \mu \mathrm{s}$, number of pulse per second $=3$, average power $=3 \mathrm{~W}$, air flow rate $=2.5 \times 10^{5} \mathrm{~mm}^{3} / \mathrm{s}$, time $=20 \mathrm{sec}$.

The measured parameters are presented in Table 2 and average values of output parameters such as aspect ratio (depth/diameter) thickness of heat affected zone and corresponding $\mathrm{S} / \mathrm{N}$ ratio (17 Taguchi's reference.) values are calculated and presented in Table 3.

Table 2 Measured hole parameters.

\begin{tabular}{|c|c|c|c|c|c|c|c|c|c|}
\hline$\frac{\dot{0}}{\dot{z}}$ & 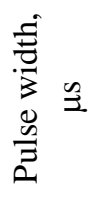 & 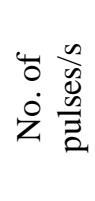 & 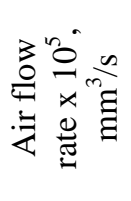 & 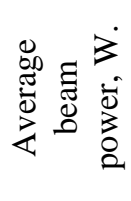 & $\stackrel{\infty}{\stackrel{\Xi}{\Xi}}$ & 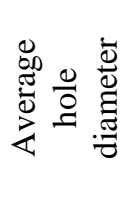 & 站育 & 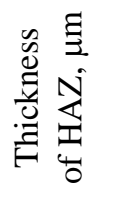 & 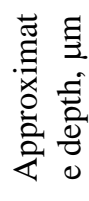 \\
\hline 1 & 500 & 1 & 0.8 & 0.8 & 20 & 322 & 18 & 59.25 & 1360 \\
\hline 2 & 500 & 2 & 2.5 & 1.1 & 20 & 321 & 78 & 24.50 & 1860 \\
\hline 3 & 500 & 3 & 4.1 & 1.7 & 20 & 258 & 07 & 24.25 & 1110 \\
\hline 4 & 700 & 1 & 2.5 & 1.6 & 20 & 212 & 79 & 72.00 & 1310 \\
\hline 5 & 700 & 2 & 4.1 & 1.8 & 20 & 146 & 43 & 82.25 & 853 \\
\hline 6 & 700 & 3 & 0.8 & 2.0 & 20 & 194 & 03 & 33.00 & 110 \\
\hline 7 & 900 & 1 & 4.1 & 1.5 & 20 & 188.5 & 56 & 42.50 & 1160 \\
\hline 8 & 900 & 2 & 0.8 & 2.5 & 20 & 161.5 & 50 & 26.00 & 847 \\
\hline 9 & 900 & 3 & 2.5 & 3.0 & 20 & 142 & 22 & 31.00 & 1070 \\
\hline
\end{tabular}

Table 3 : Calculated hole parameters

\begin{tabular}{|c|c|c|c|c|c|c|c|c|c|}
\hline $\begin{array}{l}\stackrel{0}{z} \\
\dot{n}\end{array}$ & 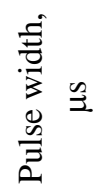 & 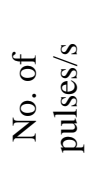 & 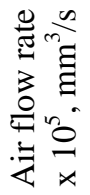 & 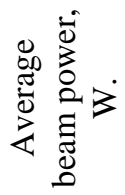 & $\underset{\Xi}{\stackrel{\infty}{\Xi}}$ & 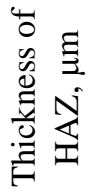 & 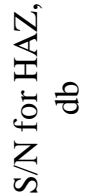 & 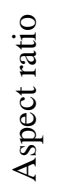 & 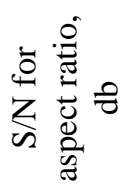 \\
\hline
\end{tabular}


IOSR Journal of Engineering

Mar. 2012, Vol. 2 (3) pp: 382-388

\begin{tabular}{|c|c|c|c|c|c|c|c|c|c|}
\hline 1 & 500 & 1 & 0.8 & 0.8 & 20 & 59.25 & -35.487 & 4.2 & 12.46 \\
\hline 2 & 500 & 2 & 2.5 & 1.1 & 20 & 24.50 & -28.036 & 5.79 & 15.25 \\
\hline 3 & 500 & 3 & 4.1 & 1.7 & 20 & 24.25 & -27.857 & 4.30 & 12.67 \\
\hline 4 & 700 & 1 & 2.5 & 1.6 & 20 & 72 & -37.8 & 6.18 & 15.82 \\
\hline 5 & 700 & 2 & 4.1 & 1.8 & 20 & 82.25 & -38.440 & 5.84 & 14.29 \\
\hline 6 & 700 & 3 & 0.8 & 2.0 & 20 & 33 & -30.436 & 5.67 & 15.07 \\
\hline 7 & 900 & 1 & 4.1 & 1.5 & 20 & 42.50 & -36.160 & 6.15 & 15.78 \\
\hline 8 & 900 & 2 & 0.8 & 2.5 & 20 & 26.00 & -30.358 & 5.25 & 14.40 \\
\hline 9 & 900 & 3 & 2.5 & 3.0 & 20 & 31.00 & -29.987 & 7.54 & 08.77 \\
\hline
\end{tabular}

\section{Discussions}

Using the above results graphs were plotted as shown in Fig. 5 and 6 .
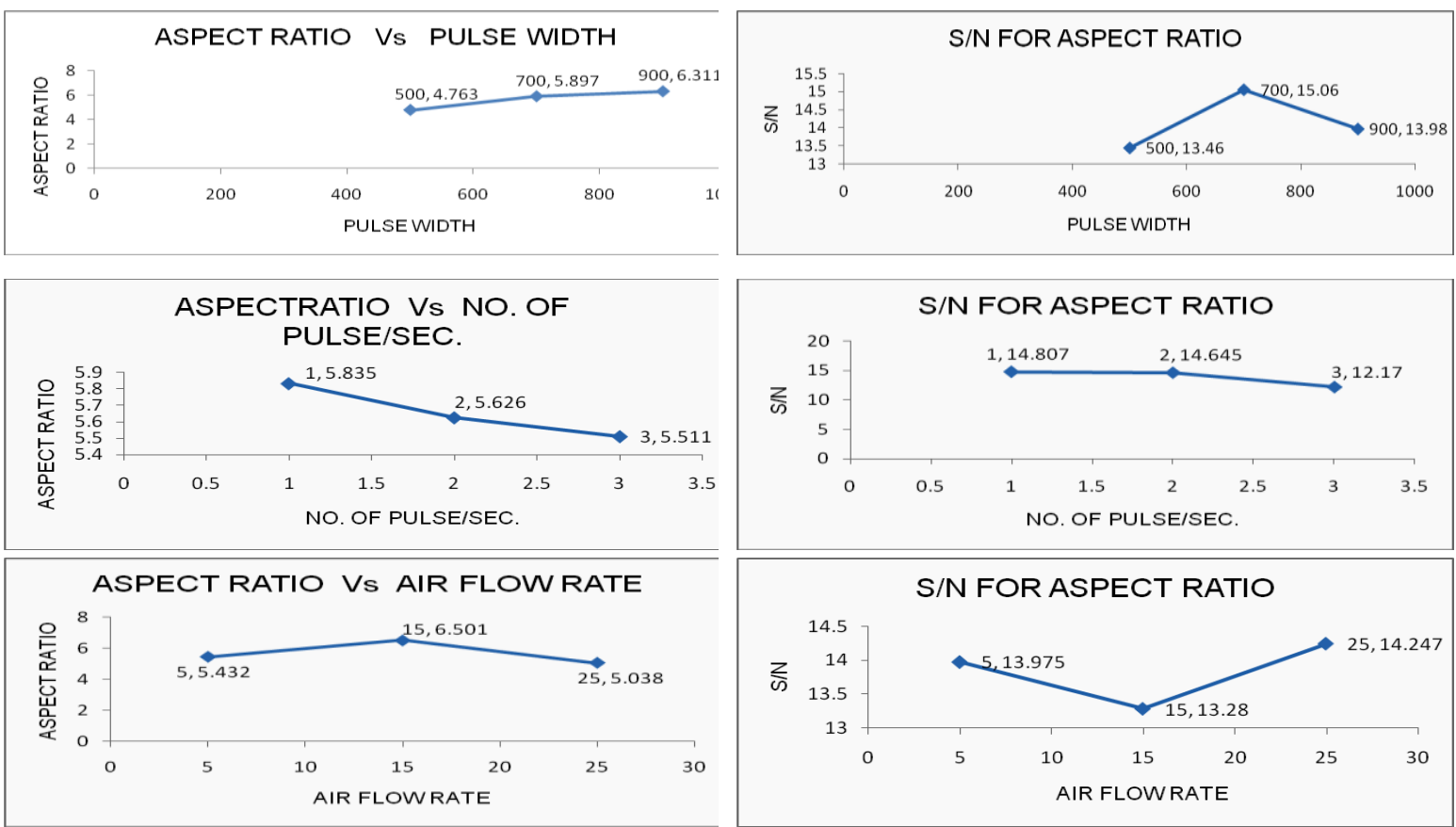

Fig. 5 A set of graphs for optimum aspect ratio. 

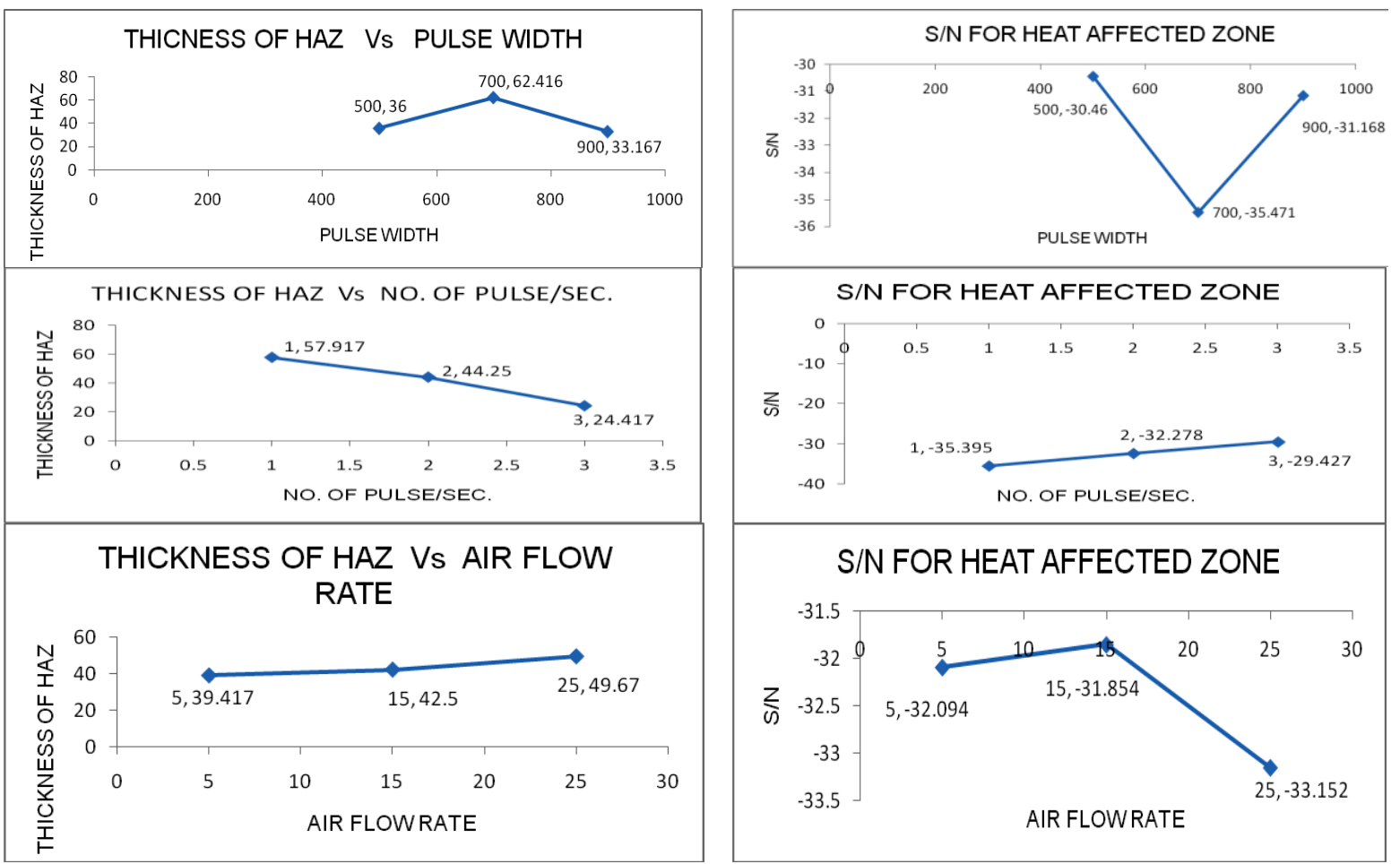

Fig. 6 A set of graphs for optimum HAZ.

It is observed from the set of figures 5 that the maximum aspect ratio (depth/average hole diameter) was obtained at high value of pulse width $(900 \mu \mathrm{s})$ and minimum pulse frequency (1pulse/sec) and medium air flow rate. At high pulse width, the heat diffusion in the depth directions is higher compared to radial (lateral) direction. Minimum pulse frequency reduces the heat diffusion in the lateral directions and hence diameter is less resulting in high aspect ratio.

Similarly it is observed from figure 6 that the thickness of heat affected zone is minimum for high pulse width $(900 \mu \mathrm{s})$ and high pulse frequency with low air flow rate. The reason for low heat affected zone at high pulse width is stated above. At high pulse frequency, the heat cannot get more time to diffuse in the lateral directions due to obstruction made by ejection of vapour and molten metal coming out of the hole. At low air flow rate, the oxidizing effect is low as a result of which lateral damage is also low.

\section{Conclusions}

The following conclusions are obtained from the present study:-

i. A pulse width of $900 \mu \mathrm{s}$ is suitable for getting a micro-hole with least heat affected zone and with high aspect ratio.

ii. A pulse frequency of 3 per second is suitable for getting least heat affected zone whereas; pulse frequency of 1 is suitable for getting high aspect ratio.

iii. The amount of air flow has negligible effect on the value of HAZ and aspect ratio but low to medium rate (0.8$2.5 \times 10^{5} \mathrm{~mm}^{3} / \mathrm{s}$ ) of air flow is suitable for laser drilling as observed from the experimental results.

iv. Best circular hole is obtained at a pulse width of $700 \mu$ s, with pulse frequency of 3 per second and at low air flow rate (i.e. $0.8 \times 10^{5} \mathrm{~mm}^{3} / \mathrm{s}$ )

v. From the SEM photographs it was found that for a pulse width of $700 \mu$ s and pulse frequency of 3 per second and with low air flow rate the deposition of spatter is minimum.

vi. High aspect ratio with minimum heat affected zone cannot be achieved simultaneously as observed from these experiments. Therefore, a compromise has to be made between these two parameters.

vii. Optimum parameters are reported by conducting only 9 experiments using Taguchi's technique where both costs and times are saved.

\section{References}

1. Mohammed N. "Laser Percussion Drilling of Aerospace Material using high peak power fiber delivered lamp pumped pulsed Nd-YAG laser” Laser Institute of America, 2006, 148-156.

2. Chrysslouris G., "Laser Machining, Theory and Practice”, Springer - Verlag, 1991, New York. 
3. Rana J, Suar S. and Bhoi R.K., "Optimization of process parameters in laser drilling using Taguchi Technique", Manufacturing Technology and Research, Vol. 4 (1-2), January to June 2008, pp. 70-78.

4. Voisey K. T., Kudesia S.S., Roden W.S.O., Hand D.P., Jones J.D.C. and Clyne T.W., "Melt Ejection during Laser Drilling of Metals", Materials Science and Engineering, A : Structural Materials : Properties, Microstructure and Processing, A 356 (1-2), 2003, pp 414-424

5. Mishra P. K., "Nonconventional Machining”, Narosa Pub. House, 1997, New Delhi.

6. Low D.K.Y, Li L and Byrd P.J., "Spatter Prevention during Laser Drilling of Selected Aerospace Materials", Journal of Materials Processing Technology, Vol 139 (1-3)], 2003, pp 71-76.

7. Low D.K.Y., Li L. and Corfe A.G., "Characteristics of spatter formation under the effects of different laser parameters during laser drilling”, Journal of materials processing Technolgy, Vol. 118 (1-3), 2001, pp179-186.

8. Zhu X, Choi J.W., Cole R. and Ahn C.H., "A new micromachining technique using a mixed mode ablation approach", IEEE International conference on MEM system, NV, USA, Jan. 20-24, pp. 152-155.

9. Gue D., Cai K. Yang J. and Huang Y., "Spatter free laser drilling of alumina ceramics based on gel casting technology", Journal of European Ceramic Society, Vol 23(8), 2003, pp.1263-1267.

10. Wang X.Y., Ng G.K.L., Liu Z. Li L. And Bradley L., "EPMA micro analysis of recast layers produced during laser drilling of type 305 stainless steel”, "Thin solid films", Vol. 453-454, 2004 pp. 84-88.

11. Schoonderbeek, Biesheuvel C.A., Hofstra R.M. Boller K.J. and Meijer J., "The influence of the pulse length on the drilling of metals with an excimer laser", Journal of laser applications, Vol. 16(2), 2004 pp.85-91.

12. Nedialkov N.N., Atanasov P.A., Sawczak M. and Sliinski G., "Laser drilling of AlN ceramics using nanosecond pulses, "Proceedings of SPIE - The International Society for Optical Engineering, 2005, 5777.

13. Nedialkov N.N., Sawczak M., Jadraque M., Atanasov P.A., Martin M., Nnd Sliwinski G., "Effect of Laser drilling on a surface and material properties of AlN ceramics.

14. Campbell B.R., Semak V.V. and Thomas J.G., “An analysis of ultrashort pulse laser micromachining parameters for optimization of shallow hole drilling”, Proceedings of SPIE-The International Society for Optical Engineering, 2006, 6261.

15. Naeem M., "Laser percussion drilling of aerospace materials using high peak power fibre delivered lamp-pumped pulsed Nd-YAG laser," Laser Institute of America, Vol. 99 2006, pp. 148-156

16. Rana J., Panda D.K. and Bhoi R.K., "Laser Drilling - A Qualitative Approach, 20 ${ }^{\text {th }}$ AIMTDR, BIT, Mesra, Ranchi, 13-15 December 2003, pp.343-348.

17. Taguchi G., "Introduction to Quality Engineering”, UNI Pub., 1986, New York. 